\title{
From the National Perinatal Information Center: The Role of Neonatology within AIM: The Alliance for Innovation on Maternal Health (AIM)
}

Elizabeth Rochin, PhD, RN, NE-BC

The National Perinatal Information Center (NPIC) is driven by data, collaboration and research to strengthen, connect and empower our shared purpose of improving patient care.

For over 30 years, NPIC has worked with hospitals, public and private entities, patient safety organizations, insurers and researchers to collect and interpret the data that drives better outcomes for mothers and newborns.

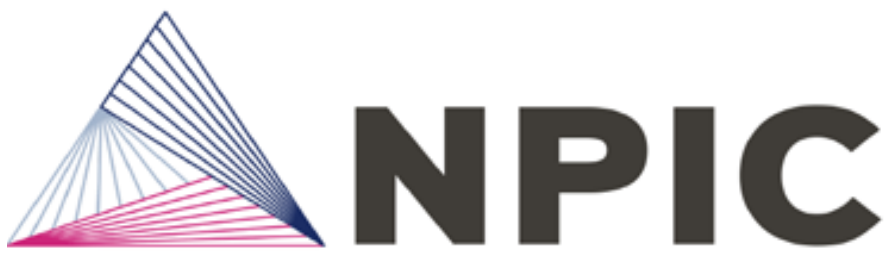

National Perinatal Information Center

\section{"AIM works through state-led hospital and healthcare teams to implement evidence-based patient care "bundles" to standardize maternal care and drive optimal outcomes. As of this publication date, there are 38 states currently enrolled in AIM."}

The Alliance for Innovation on Maternal Health (AIM) was established in 2014 through the US Department of Health and Human Services' Health Resources and Services Administration (HRSA) Maternal and Child Health Bureau (MCHB). AIM is a recognized national data-driven maternal safety and quality improvement initiative based on multidisciplinary consensus-based practices to improving maternal safety and outcomes. AIM works through state-led hospital and healthcare teams to implement evidencebased patient care "bundles" to standardize maternal care and drive optimal outcomes. As of this publication date, there are 38 states currently enrolled in AIM. Currently, the approved AIM Patient Care Bundles consist of:

- Maternal Venous Thromboembolism

- Obstetric Care for Women with Opioid Use Disorder (OUD)

- Obstetric Hemorrhage

- Postpartum Care Basics for Maternal Safety

- Safe Reduction of Primary Cesarean Section

- Severe Hypertension in Pregnancy

- Reduction of Peripartum Racial/Ethnic Disparities (being rolled into all bundles in 2021)
The National Perinatal Information Center (NPIC) sits on the AIM Executive Committee. The Executive Committee assists the AIM leadership team in reviewing state applications and assists in reviewing state data plans as well as offering overall support of the mission and vision of AIM. Currently, the state application does not request a neonatology resource as a member of the Clinical Champions team. However, the inclusion of neonatology will be a critical source of expertise and support as states continue to and begin implementation of the Obstetric Care for Women with Opioid Use Disorder bundle, one of the most frequently implemented bundles at this time.

The AIM Opioid Use Disorder bundle includes the following multidisciplinary resource recommendations that specifically require the expertise and guidance from neonatologists, particularly in regions that experience a high NICU admission rate associated with NAS:

- Provide staff-wide (clinical and non-clinical staff) education on SUDs.

- Emphasize that SUDs are chronic medical conditions that can be treated.

- Emphasize that stigma, bias and discrimination negatively impact pregnant women with OUD and their ability to receive high quality care.

- Establish specific prenatal, intrapartum and postpartum clinical pathways for women with OUD that incorporate care coordination among multiple providers

- Know federal (Child Abuse Prevention Treatment Act - CAPTA), state and county reporting guidelines for substance-exposed infants

Between 2014 and 2016, the opioid epidemic and discussions regarding its impact on pregnancy became a matter of public policy. Krans \& Patrick (2016) described the proportion of pregnant women admitted to substance abuse treatment facilities that reported a history of prescription opioid abuse increased from $2 \%$ to $28 \%$ between the years of 1992 to 2012. The opioid epidemic crosses all racial, ethnic, socioeconomic and geographic boundaries, further highlighting the importance of universal screening and education at all points of a woman's reproductive lifespan, which is respectful and neutral in its application.

The opioid epidemic has continued to expand and the incidence of 


\section{$\triangle$ NPIC}

Perinatal Center Database

NEONATAL ABSTINENCE SYNDROME (NAS) TREND*

Q1 2018 - Q4 2019

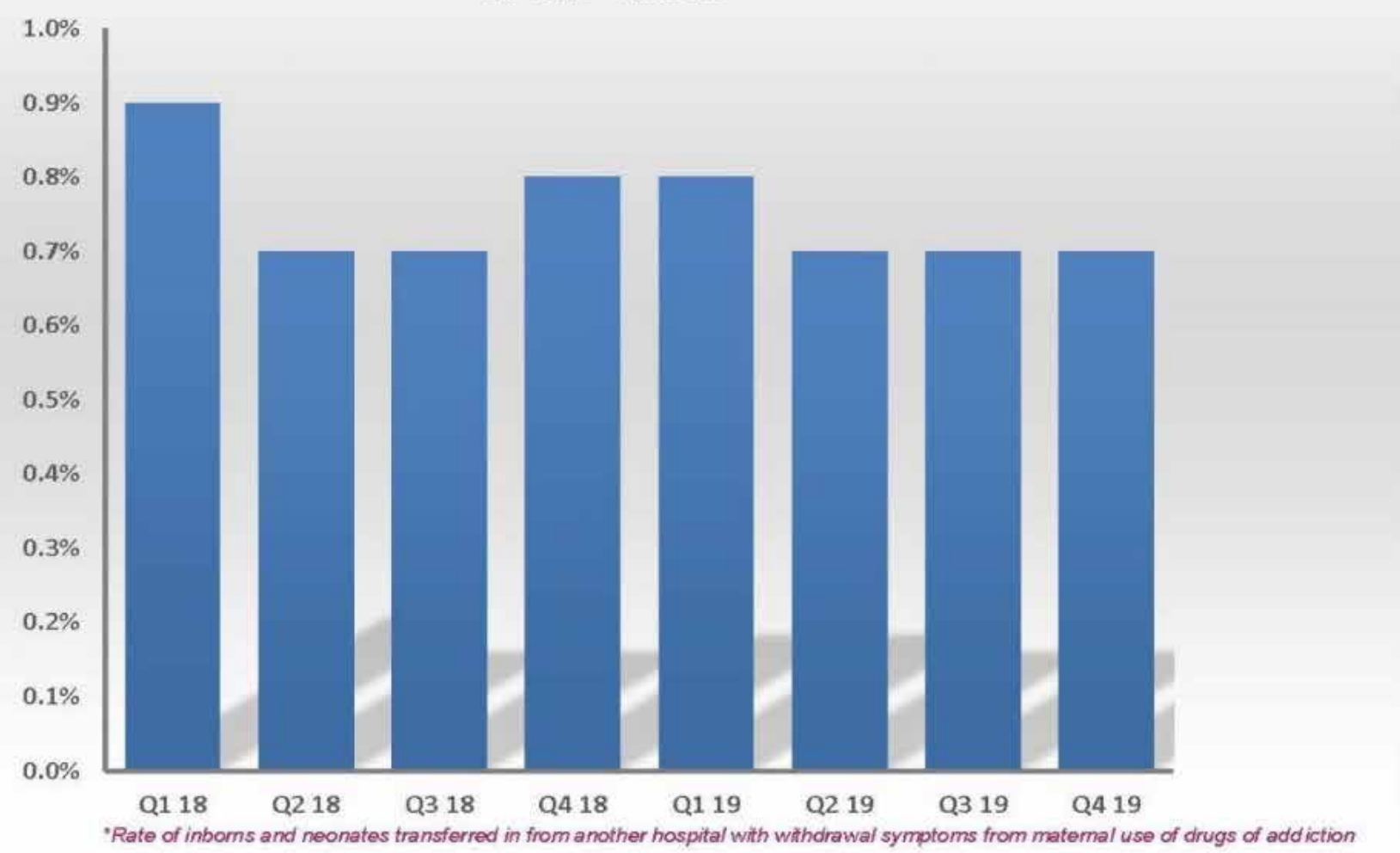

(9) NPIC 2020

'Rate of inborns and neonates transferred in from another hospital with withdrawal symptoms from matermal use of drugs of addiction

postnatal withdrawal or neonatal abstinence syndrome (NAS) has also expanded, with current estimates for infants in the US with NAS ranging between 2.6 per 1,000 births to 16.2 per 1,000 births (Hudson et al, 2019). With the increasing emphasis on keeping mothers and at-risk newborns together as a dyad during the immediate postpartum period, it is important to recognize that the most acute care for NAS will take place in the NICU. Ensuring and mobilizing an approach that keeps the mother and family involved in the care of the newborn during a NICU admission requires a strong multidisciplinary team, and added to that the complexity of NAS (including the stigma that exists for women and birthing people with OUD throughout healthcare) requires a unique and neutral approach. These are the conversations that occur within AIM teams and require the expertise and guidance from all parties, including neonatologists and neonatal practitioners, committed to the improvement of the lives of women and newborns experiencing OUD and NAS, respectively.

NPIC recognizes the importance of NAS reporting and tracking for quality and programmatic improvement and has an established online, interactive database (CAIRN: Custom Analytic Interactive Reporting Network) used for NAS metric analysis, in addition to other descriptive and outcome metrics. Based on the NPIC 20182019 aggregate data, the rate of NAS (NPIC uses the AIM definition) was between $0.7 \%$ to $0.9 \%$, with most of 2018 and 2019 hovering around $0.7 \%$.

In addition, the NPIC CAIRN platform also reports Medicaid quartiles, and there are notable differences that occur with NAS admissions and the higher Medicaid quartiles (Medicaid $\leq 22$; Medicaid 22.01 - 38.91; Medicaid 38.92 - 49.7; Medicaid $\geq 49.71$ ). More than $80 \%$ of newborns experiencing NAS have their care paid for by Medicaid (Centers for Medicare and Medicaid Services, 2018;
Winkelman et al, 2018).

While the majority of the work within AIM focuses on the mother, it is important to recognize the neonatal and pediatric contributions that will be required to successfully implement the AIM Opioid Use Disorder Bundle. Data collection and interpretation will be key to better understanding of those PDSA initiatives that are successful or require re-evaluation and retooling. Integration of a robust multidisciplinary and interdisciplinary team approach within the AIM Opioid Use Disorder bundle at the outset will provide a strong foundation for the woman and her family to navigate this chronic illness with a committed and compassionate team.

"While the majority of the work within AlM focuses on the mother, it is important to recognize the neonatal and pediatric contributions that will be required to successfully implement the AlM Opioid Use Disorder Bundle. Data collection and interpretation will be key to better understanding of those PDSA initiatives that are successful or require re-evaluation and retooling." 


\section{$\triangle N P I C$}

(c) NPIC 2020

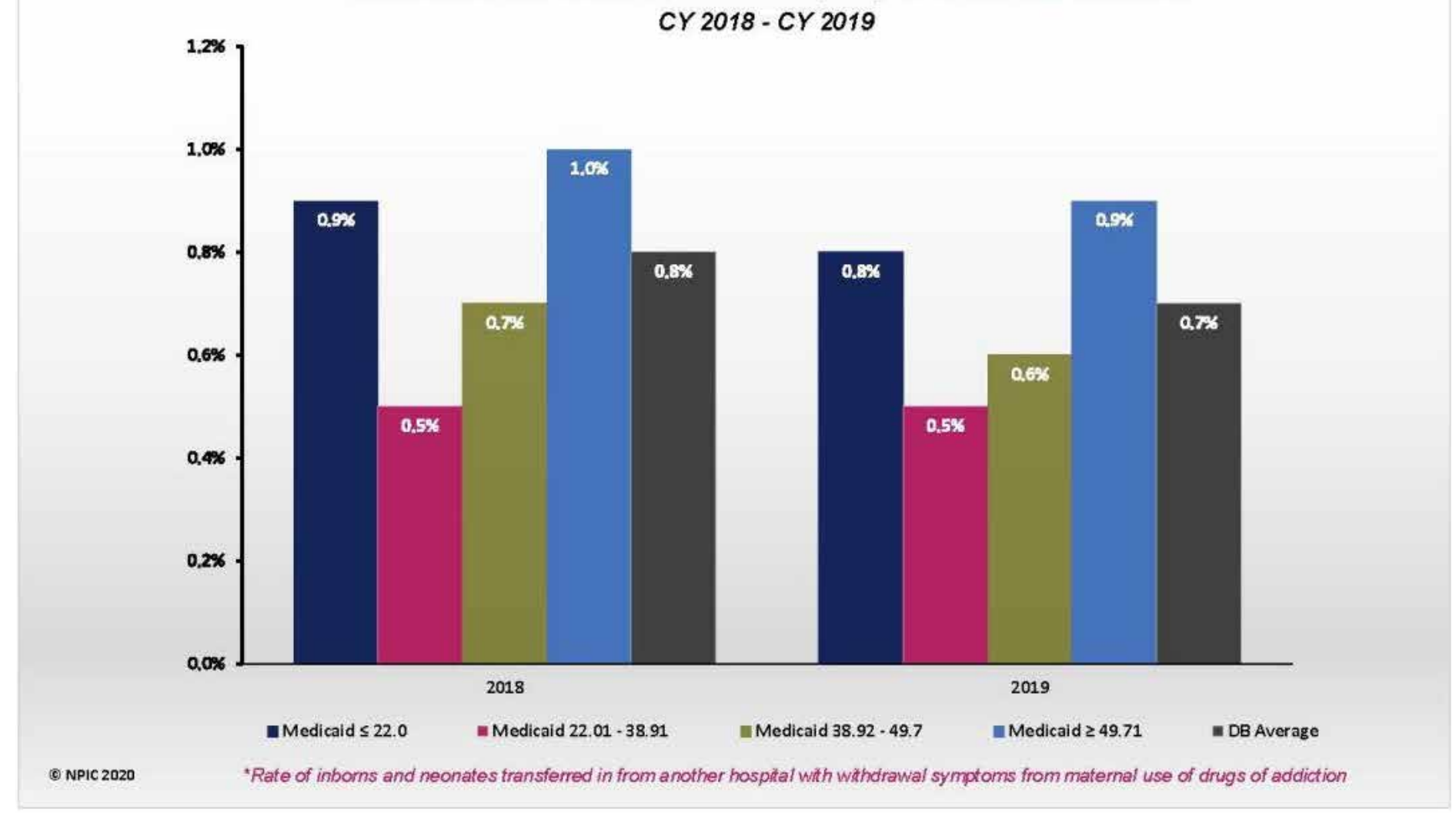

Perinatal Center Database

NEONATAL ABSTINENCE SYNDROME* (NAS) BY MEDICAID QUARTILE

CY 2018 - CY 2019

\section{References}

Centers for Medicare and Medicaid Services. (2018). https://www. cms.gov/newsroom/press-releases/cms-leverages-medicaid-program-combat-opioid-crisis-0

Hudson, J., Charron, E., Bedi, J., Dikes, L., Mayo, R. \& Sherrill, W.W. (2019). Managing abstinence in newborns (MAiN): Redesigning NAS care for the mother/baby unit. Healthcare, 8(1), https://doi.org/10.1016/j.hjdsi.2019.100404

Krans, E.E. \& Patrick, S.W. (2016). Opioid use disorder in pregnancy: Health policy and practice in the midst of an epidemic. Obstetrics \& Gynecology, 128(1), 4-10. doi: $10.1097 /$ AOG.0000000000001446

Spence, K., Boedeker, R., Harhausen, M., Kaushal, G., Buchanan, P. \& Josephsen, J. (2020). Avoiding NICU transfers for newborns with neonatal opioid withdrawal syndrome (NOWS): A quality improvement initiative to manage NOWS on the mother-baby unit. Journal of Addiction Medicine, 14(5), 401408. https://doi: 10.1097/ADM.0000000000000607

Winkelman, T.N., Villapiano,. N., Kozhimannil, K.B., Davis, M.M. \& Patrick, S.W. (2018). Incidence and costs of neonatal abstinence syndrome among infants with Medicaid: 2004-2014. Pediatrics, 141(4), doi: 10.1542/peds.2017-3520
The author has no conflicts of interests to disclose.

\section{NT}

\section{Corresponding Author:}

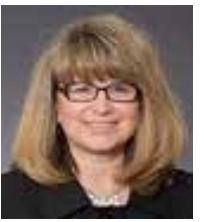

Elizabeth Rochin, PhD, RN, NE-BC

President

National Perinatal Information Center

225 Chapman St. Suite 200

Providence, RI 02905

401-274-0650

Email: inquiry@npic.org

NEONATOLOGY TODAY is interested in publishing manuscripts from Neonatologists, Fellows, NNPs and those involved in caring for neonates on case studies, research results, hospital news, meeting announcements, and other pertinent topics.

Please submit your manuscript to: LomaLindaPublishingCompany@gmail.com 